\title{
Rendimiento, motivación y satisfacción académica, ¿una relación de tres?
}

Laura Hernández Vaquero. Universidad de Granada

Clara Isabel Martín Fernández. Universidad de Granada

Gloria Lorite Ruiz. Universidad de Granada

Paula Granados Bautista. Universidad de Granada

Recepción: 26.02.2018 | Aceptado: 5.03.2018

Correspondencia a través de ORCID: Laura Hernández Vaquero iD 0000-0002-4642-7312

Citar: Hernandez, L., Fernandez, C., Lorite, G. y Granados, P. (2018). Rendimiento, motivación y satisfacción académica, ¿una relación de tres? ReiDoCrea,7, 92-97.

Resumen: La literatura nos muestra que la motivación, el rendimiento y la satisfacción son variables con una estrecha correlación que ha sido explicada por diversas teorías y modelos del aprendizaje desarrollados desde los años 80 . El objetivo de este trabajo fue realizar un estudio correlacional sobre la motivación, el rendimiento y la satisfacción académica de estudiantes de la Universidad de Granada. Se utilizó una muestra de 33 estudiantes con edad entre los 18 y 24 años. De esta forma, se analizó la relación existente entre motivación y rendimiento y entre éste último y la satisfacción. Para medir el rendimiento se utilizaron dos vías, la nota media y los estilos de aprendizaje. Además se estudió la posibilidad de que hubiera diferencias en satisfacción en función de la elección voluntaria o impuesta del grado cursado. Los resultados mostraron que el rendimiento medido a través de la nota media se relaciona de forma positiva con la motivación y con la satisfacción.

Palabras clave: Motivación | Satisfacción

Performance, Motivation and Academic Satisfaction: A Three-Sided Relationship?

\begin{abstract}
The literature shows that motivation, performance and satisfaction are variables with a close correlation that has been explained by diverse learning theories and models developed since the $80 \mathrm{~s}$. The objective of this investigation was to carry out a correlational study on the motivation, performance and academic satisfaction of students of the University of Granada. The sample consisted of 33 students between the ages of 18 and 24. In this way, the relationship between motivation and performance and between the latter and satisfaction was analyzed. Performance was measured in two ways, the average grade and learning styles. In addition, the possibility of differences in satisfaction based on the voluntary or imposed choice of degree was studied. The results showed that the performance measured through the mean score is positively related to motivation and satisfaction.
\end{abstract}

Keywords: Motivation | Satisfaction

\section{Introducción}

¿Qué relación existe entre la motivación, el rendimiento y la satisfacción en el ámbito académico? Este enfoque ha sido explicado por diversas teorías y modelos del aprendizaje desarrollados desde los años 80 (Weinert, 1987; Zimmerman y Schunk, 1989, citado en Roces,González-Torres y Tourón, 1997).

La literatura nos muestra que la satisfacción es una variable con una estrecha correlación con el rendimiento, al igual que la motivación. La relación entre satisfacción, motivación y rendimiento es de carácter dinámico pudiendo influir la satisfacción y motivación de forma positiva o negativa sobre el rendimiento del individuo (Gîlmeanu, 2015).

El rendimiento académico se puede medir observando los resultados traducidos en calificaciones que se obtienen en exámenes. Blanz (2014) señala que es el producto de la motivación, la voluntad, la capacidad y la influencia del componente social. De acuerdo con Livengood (1992) el rendimiento académico es una variable que puede 
ayudar a determinar si el estudiante concluirá y obtendrá su título universitario; lo que podríamos considerar una meta de logro en relación con la motivación.

Torres (2014) destaca que el rendimiento académico está asociado con el conocimiento de los estilos de aprendizaje por parte de alumnos y docentes. Con este conocimiento los profesores podrían adaptar mejor sus clases a las capacidades de los alumnos. Sin embargo, se debe destacar que el buen rendimiento es el resultado de la administración del tiempo y de las habilidades para el estudio, el apoyo de los compañeros, la familia, no solo de la ayuda de los profesores (Mamiseishvili, 2012).

Por otro lado, la motivación es un tema muy tratado en Psicología de las Organizaciones porque define las razones de por qué se llevan a cabo las acciones (Mitchell \&Daniels, 2003). Este constructo ha sido definido por muchos autores y perspectivas y se ha relacionado con otros constructos como la satisfacción y el rendimientos en ámbitos laborales y académicos; centro de nuestra investigación. La motivación hace referencia a los procesos psicológicos que direccionan, dan energía y sustentan las acciones (Latham y Pinder, 2005). Destacamos la división entre motivación intrínseca y extrínseca. La motivación intrínseca es el estado subjetivo que mueve la realización de una actividad sin ninguna recompensa externa. En el ámbito de la educación, la curiosidad y el interés son los estímulos intrínsecos fundamentales.

En la motivación extrínseca se llevan a cabo acciones esperando una recompensa. En el ámbito académico, las recompensas que favorecen la motivación extrínseca son las alabanzas, las buenas notas o los premios (Reeve, 1994).

Respecto a la relación entre motivación y satisfacción, hay autores que defienden que existe una relación lineal unidireccional entre ambos constructos, de forma que la satisfacción puede ser causa o consecuencia de la motivación. Pero esta perspectiva está incompleta porque no explica claramente el fenómeno que se analiza. Por el contrario, la perspectiva de Zlate parece especialmente adecuada al relacionar la motivación y la satisfacción con estas tres afirmaciones: a. La satisfacción/insatisfacción es un indicador de motivación, b. La motivación y la satisfacción son causas y consecuencias la una de la otra, c. Ambas se reflejan en el rendimiento de un sujeto, pudiendo afectar a este de forma negativa o positiva (Gîlmeanu, 2015).

Finalmente, la satisfacción estudiantil se ha definido como el grado de congruencia entre las expectativas previas de los estudiantes y los resultados obtenidos en el proceso de aprendizaje (Allen, Omori, Burrell, Mabry y Timmerman, 2013; Moore y Shelton, 2014). Numerosos estudios demuestran que la alta satisfacción de los universitarios correlaciona positivamente con un buen rendimiento y progreso académico (Medrano y Pérez, 2010). La satisfacción académica se vincula al bienestar general de haber logrado las metas planteadas; la autorrealización (FernándezAbascal, et al., 2011).

A través del estudio estadístico llevado a cabo por González-Peiteado, Pino-Juste y Penado se ha podido comprobar que la mayor parte de los estudiantes se sienten satisfechos con su vida universitaria, manifestando un alto grado de bienestar con las diferentes variables analizadas. Los resultados muestran que la satisfacción global del alumno universitario repercute en su calidad de vida global y en su identificación con la institución (Blázquez et al., 2013). 


\section{Objetivos o hipótesis}

Teniendo en cuenta este marco teórico, el objetivo de nuestra investigación es estudiar la relación existente entre la motivación y el rendimiento académico de estudiantes universitarios. A su vez, analizaremos la relación entre el rendimiento académico y la satisfacción con el grado que cursen. El rendimiento se medirá a través de la nota media y de los estilos de aprendizaje. Como factor influyente en la satisfacción consideramos la elección libre o impuesta del grado según si fue o no su primera opción.

Las hipótesis formuladas son las siguientes.

- H1: La motivación correlacionará de forma positiva con el rendimiento medido a través de la nota media y los estilos de aprendizaje.

- H2: El rendimiento medido a través de la nota media y de los estilos de aprendizaje correlacionará de forma positiva con la satisfacción.

- H3: Habrá diferencias en satisfacción en función de la elección voluntaria o no del grado.

\section{Métodos}

\section{Participantes}

En el estudio se utilizó una muestra de 33 estudiantes de la Universidad de Granada (12 hombres y 21 mujeres). El rango de edad variaba entre los 18 y 24 años $(M=19,93$; $D T=1,028)$. Los participantes fueron alumnos de distintos grados y cursos.

\section{Instrumentos}

Los instrumentos utilizados para medir las variables estudiadas contenían escalas de tipo Likert en su formato de respuesta. Fueron los siguientes: en primer lugar, para medir el rendimiento académico se formuló una pregunta directa para conocer la nota media del participante. Además se utilizó para medir los estilos de aprendizaje el StudyProcessQuestionnaire (R-SPQ-2F) de Biggs y Kember (2001) adaptado.

En cuanto a la motivación el instrumento utilizado fue la Escala de Motivación del Aprendizaje y Estilos Atribucionales: Escala CEAP48 de Barca, Porto, Santorum, Brenlla, Morán y Barca (2005).

Por último, para evaluar la variable satisfacción, empleamos el Cuestionario de Satisfacción con la Experiencia Universitaria (CSEU) de Kember y Leung (2005) validado en su versión española por González-Peiteado, Pino-Juste y Penado-Abilleira (2016). Para conocer si influye en las variables estudiadas el tipo de elección del grado, se formuló también una pregunta directa sobre esta cuestión.

\section{Procedimiento}

En primer lugar, se pidió a los participantes que leyeran detenidamente las instrucciones y el consentimiento informado. Una vez aceptada la participación, se le proporcionaron los cuestionarios a rellenar. El tiempo de aplicación de los cuestionarios fue de 10 minutos aproximadamente por cada uno. 
Las primeras cuestiones que se les presentaron fueron acerca de la nota media y la elección del grado. El interés de esta última era conocer si el participante eligió el grado que cursa en la actualidad como primera opción o por el contrario la elección se llevó a cabo por otros motivos. Seguidamente se contestaron los instrumentos descritos anteriormente. El primero fue cuestionario de estilos de aprendizaje, el segundo el de motivación académica y el último el de satisfacción académica.

\section{Análisis de datos}

El análisis de datos se realizó empleando el programa estadístico SPSS. En primer lugar, se llevó a cabo un análisis descriptivo de los datos obtenidos en satisfacción, motivación, nota media y estilos de aprendizaje (media y desviación típica). Seguidamente, se analizaron empleando correlaciones bivariadas entre los pares de variables. También se realizó una T de Student para muestras independientes, con la variable independiente elección de carrera y la variable dependiente satisfacción.

\section{Resultados}

Se analizaron las variables estudiadas motivación, satisfacción y rendimiento, éste último medido a través de la nota media y de los Estilos de Aprendizaje, obteniéndose la media y desviación típica de cada variable (Ver Tabla 1).

\begin{tabular}{|c|c|c|}
\hline \multicolumn{3}{|c|}{ TABLA 1. Medias y Desviaciones típicas de cada variable } \\
\hline & Media & Desviación típica \\
\hline Motivación & 3,8706 & 0,61029 \\
\hline Satisfacción & 3,5085 & 0,68546 \\
\hline Nota media & 7,5673 & 0,90828 \\
\hline Estilo Aprendizaje & 2,5636 & 0,35582 \\
\hline
\end{tabular}

Para comprobar las cuatro primeras hipótesis formuladas, se analizaron los datos con correlaciones bivariadas, obteniendo el Coeficiente de Correlación de Pearson de cada relación establecida (Ver Tabla 2). En primer lugar, encontramos que la correlación entre motivación y rendimiento medido a través de la nota media $(\mathrm{H} 1)$ es significativa y positiva. Sin embargo, la correlación entre motivación y rendimiento medido a través de estilos de aprendizaje ( $\mathrm{H} 2)$ no es significativa.

Por otro lado, obtuvimos que la correlación entre el rendimiento medido a través de la nota media y la satisfacción $(\mathrm{H} 3)$ es significativa y positiva. De igual forma, la correlación entre el rendimiento medido a través de los estilos de aprendizaje y la satisfacción (H4) no es significativa.

\begin{tabular}{|c|c|c|c|c|}
\hline & Motivación & Satisfacción & Nota media & $\begin{array}{c}\text { Estilos } \\
\text { Aprendizaje }\end{array}$ \\
\hline Motivación & & 0,159 & $0,589^{* *}$ & 0,247 \\
\hline Satisfacción & 0,159 & & $0,452^{* *}$ & $-0,17$ \\
\hline Nota media & $0,589^{* *}$ & $0,452^{* *}$ & & 0,216 \\
\hline Estilos Aprendizaje & 0,247 & $-0,17$ & 0,216 & \\
\hline
\end{tabular}

Para comprobar la quinta hipótesis, se llevó a cabo una T de Student para muestras independientes, con la variable independiente elección de carrera y la variable dependiente satisfacción. Los resultados no mostraron diferencias significativas en satisfacción en función de la elección de carrera $t(31)=1.37, p=.19$. Las medias (con 
las desviaciones típicas entre paréntesis) para la opción elegida y la no elegida fueron $3,61(0,66$ y $3,23(0,73)$, respectivamente.

\section{Discusión}

Tras el estudio, podemos afirmar que se cumplen dos de las tres hipótesis planteadas. La primera hipótesis se cumple, concluimos que la motivación correlaciona de forma positiva con el rendimiento medido a través de la nota media. Como comentábamos en la introducción, los resultados traducidos en calificaciones son una de las medidas que más nos ayudan a determinar el rendimiento académico. Nuestra segunda hipótesis se ve cumplida en cuanto a que hay correlación entre el rendimiento medido a través de la nota media y la satisfacción. Este resultado concuerda con lo que citaban Medrano y Pérez (2010), demostrando que la alta satisfacción de los universitarios correlaciona positivamente con un buen rendimiento y progreso académico. En relación a los estilos de aprendizaje, Torres (2014) asociaba el rendimiento al conocimiento de estos estilos, sin embargo, no hemos encontrado esa correlación. Las hipótesis no se ven cumplidas al no obtener resultados significativos. Lo mismo ocurre con la hipótesis número tres, no hay diferencias en satisfacción en cuanto a la elección voluntaria o no del grado. Esto puede deberse a la limitación en el número de participantes de cada condición. Hubo bastante diferencia en número de personas entre los que eligieron su carrera como primera opción y los que no lo hicieron.

En cuanto a los estilos de aprendizaje, la mayoría de los resultados se situaron en torno a valores centrales, esto es, se encontraban entre los dos estilos de aprendizaje, superficial y profundo. Creemos que al no situarse en un estilo de aprendizaje, no se ha asociado tanto con la motivación o satisfacción.

Como limitaciones, comentar que este estudio es correlacional y los resultados no son causales, no podemos afirmar la dirección en la que se influyen nuestras variables. En futuros estudios podría analizarse su relación causal.

También sería interesante para futuras investigaciones comprobar si hay diferencias en motivación, rendimiento y satisfacción en cuanto al curso que se esté estudiando o en cuanto a la carrera que se esté cursando.

\section{Referencias}

Barca, A., Barca, E., Brenlla, JC., Morán, H., Porto, A. y Santorum, R. (2005). La escala CEAP48: un instrumento de evaluación de la motivación académica y atribuciones causales para el alumnado de enseñanza secundaria y universitaria de Galicia. Revista de Psicología y Educación, 1(2), 103-136.

Fernández Rico, JE., Fernández Fernández, S., Alvarez Suárez, A. y Martinez Camblor, P. (2007). Éxito académico y satisfacción de los estudiantes con la enseñanza universitaria. RELIEVE, 13,(2), 203-214.

Gargallo López, B., Garfella Esteban, P. y Pérez Pérez, C. (2006). Enfoques de aprendizaje y rendimiento académico en estudiantes universitarios. Bordón, 58(3), 45-61.

Gîlmeanu, R. (2015). Theoretical Considerations on Motivation at he Work Place, Job Satisfaction and Individual Performance. Valahian Journal of Economic Studies, 6 (20), 69-80.
Gómez Sánchez, D., Oviedo Marin, R. y Martínez López, E. (2011). Factores que influyen en el rendimiento académico del estudiante universitario. Tecnociencia, 5(2), 90-97.

González-Peiteado, M., Pino-Juste, M. y Penado-Abilleira, M. (2017). Estudio de la satisfacción percibida por los estudiantes de la UNED con su vida universitaria. RIED. Revista Iberoamericana de Educación a Distancia, 20(1), 243-260.

Grant, A. (2008). Does Instric Motivation Fuel the Prosocial Fire? Motivational Synergy Predicting Persistence, Performance, and Productivity. Journal of Applied Psychology, 93(1), 48-58.

Klehe, U., \& Anderson, N., (2007). Working Hard and Working Smart: Motivation and Ability During Typic and Maximun Performance. Journal of Applied Psychology, 92(4), 978-992. 
Roces, C., González-Torres, MC. y Tourón, J. (1997). Expectativas de aprendizaje y de rendimiento de los alumnos universitarios. Revista de Psicología de la Educación, 22, 99123.

Xanthopoulou, D., Baker, A., Heuven, E., Demerouti, E., \& Schaufeli, U. (2008). Working in the Sky: A Diary Study on Work Engagement Among Flight Attendants. Journal of Occupational Health Psychology, 13(4), 345-356. 